\title{
1310 nm Evanescent Hybrid III-V/Si Laser Based on DVS-BCB Bonding
}

\author{
S. Stanković ${ }^{1}$, G. Roelkens ${ }^{1}$, D. Van Thourhout ${ }^{1}$, R. Jones ${ }^{2}$, M. Sysak ${ }^{2}$ and J. Heck ${ }^{2}$ \\ ${ }^{1}$ Photonics Research Group, Ghent University - imec, Sint-Pietersnieuwstraat 41, 9000 Gent, Belgium \\ ${ }^{2}$ Intel Corporation, 2200 Mission College Blvd. SC12-326, Santa Clara, CA 95054, USA \\ Authore-mail address: Stevan.Stankovic@intec.ugent.be
}

\begin{abstract}
We present an evanescently-coupled, hybrid III-V/Silicon Fabry-Perot laser based on adhesive (DVS-BCB) bonding, operating at $1310 \mathrm{~nm}$. Maximum optical power in a continuouswave regime is $3 \mathrm{~mW}$ and the threshold current density is $2.41 \mathrm{kA} / \mathrm{cm}^{2}$.

OCIS codes: (130.0130) Integrated optics; (130.3120) Integrated optics devices
\end{abstract}

\section{Introduction}

Silicon photonics is emerging as a promising technology for the fabrication of high-performance photonic integrated circuits. It is based on the silicon-on-insulator (SOI) material platform that shares its fabrication processes and tools with microelectronics. With silicon being transparent at telecommunication wavelengths, silicon photonics offers a cost-effective integration of high-speed photonic and electronic devices on a single chip. However, due to silicon's indirect bandgap, fabrication of efficient light sources in silicon photonics is still a serious challenge.

Heterogeneous integration of III-V materials and SOI waveguides is an appealing approach to address this problem. Evanescent coupling offers the most promising way to couple light generated in the III-V material into the SOI waveguide. Researchers have already demonstrated light sources based on evanescent coupling, including Fabry-Perot [1-3], DBR [4], and DFB lasers [5]. These devices were based on a molecular, plasma-assisted, wafer bonding which requires very clean, smooth and contamination-free surfaces. We believe such strict requirements make an industrial-scale fabrication based on molecular bonding a serious challenge. Alternatively, an evanescent hybrid III-V/Si laser based on selective-area metallic bonding was demonstrated [6]. Although it offers more relaxed bonding requirements, this technique requires very precise alignment of pre-fabricated III-V and SOI dies, which is a serious drawback from the aspect of high-level integration.

In this paper, we propose an alternative approach based on adhesive bonding, using the commercially available DVS-BCB polymer (BCB, for short). We present the design and fabrication procedure of a hybrid Fabry-Perot III$\mathrm{V} /$ silicon evanescent laser operating at $1310 \mathrm{~nm}$, based on a BCB bonding. The achieved results include a maximum output power of $3 \mathrm{~mW}$ in $\mathrm{CW}$ regime and a threshold current of $65 \mathrm{~mA}$.

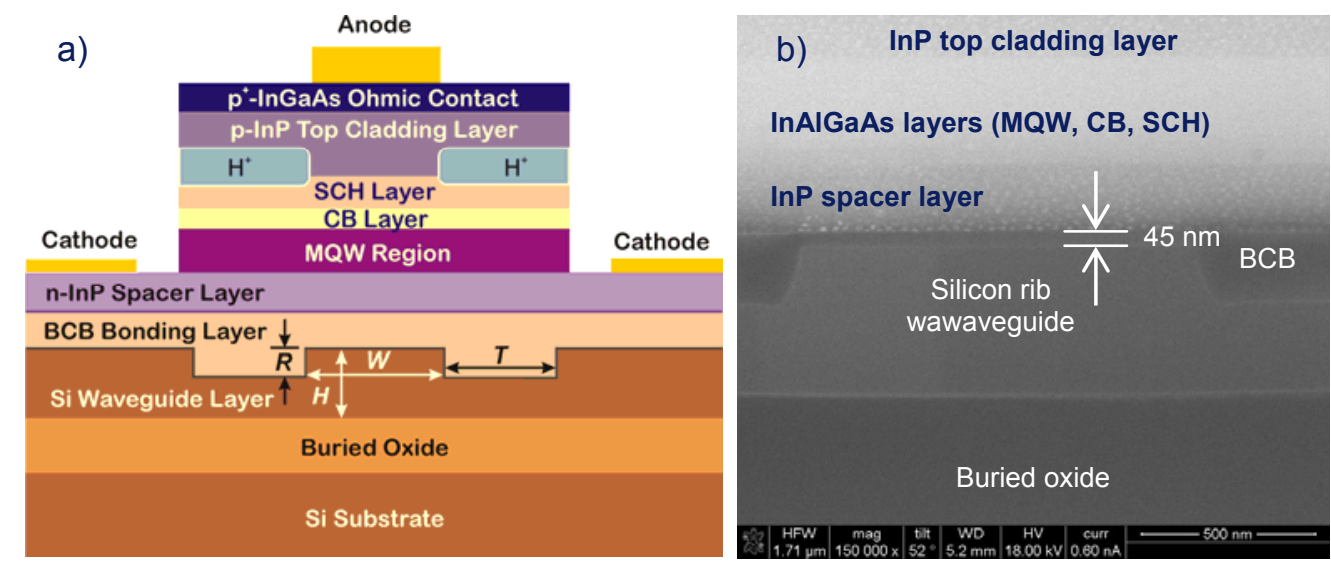

Figure 1. a) Cross-section of an evanescent, hybrid III-V/Si laser based on BCB bonding; b) SEM image of the cross-section of a III-V die bonded on top of a Si rib waveguide with a $45 \mathrm{~nm}$-thick BCB bonding layer.

\section{Hybrid laser layout}

The layout of the hybrid III-V/silicon evanescent laser is given in Figure 1a. The silicon rib waveguide is made on a SOI platform, with a $1 \mu \mathrm{m}$ thick buried oxide layer. The waveguide height and width are $\mathrm{H}=500 \mathrm{~nm}, \mathrm{~W}=0.8 \mu \mathrm{m}$, respectively. The rib etch depth is $\mathrm{R}=220 \mathrm{~nm}$ and the trench width is $\mathrm{T}=3.5 \mu \mathrm{m}$. The epitaxial III-V structure is bonded on top of the waveguide, using a BCB adhesive layer. It consists of the n-type InP spacer layer and the 16.8 


\section{IWC3.pdf}

$\mu \mathrm{m}$ wide mesa structure comprising the multiple quantum well (MQW) region (8 InGaAlAs-based QWs and 9 barriers), carrier blocking $(\mathrm{CB})$ layer, a separate confinement heterostructure $(\mathrm{SCH})$ layer, a p-type InP top cladding layer and an ohmic contact.
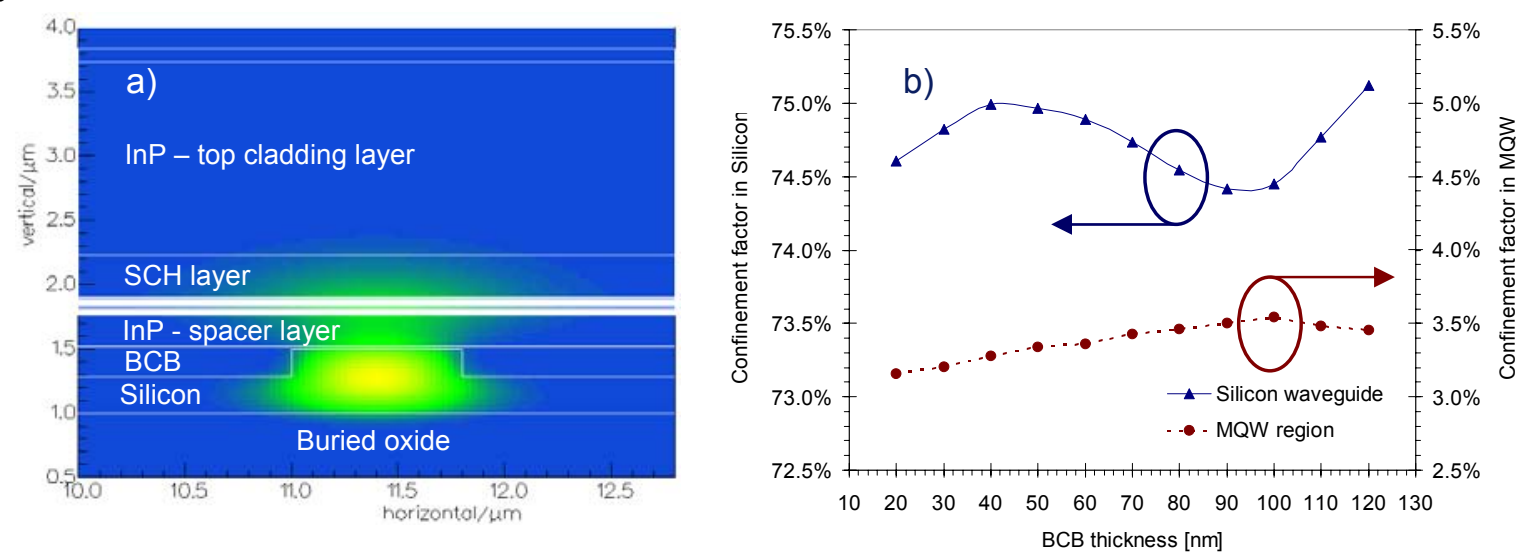

Figure 2. a) 2-D intensity plot of the fundamental hybrid mode: the primary peak is located in the Si rib waveguide and the secondary peak is in the MQW region; b) Fundamental mode confinement factors in Si waveguide $\left(\Gamma_{\mathrm{Si}}\right)$ and MQW region $\left(\Gamma_{\mathrm{MQW}}\right)$, depending on BCB layer thickness.

For efficient evanescent coupling between the modes supported by the III-V layers and the underlying SOI waveguide, the thickness of the BCB bonding layer must be sufficiently small - roughly, less than $120 \mathrm{~nm}$. The device is designed to support the fundamental hybrid optical TE mode which is predominantly confined within the $\mathrm{Si}$ waveguide, with only a fraction of the optical power within the MQW active region, as presented in Figure 2a. Based on optical simulations, the optimal thicknesses of the n-type spacer layer and the SCH layer were found to be $240 \mathrm{~nm}$ and $325 \mathrm{~nm}$, respectively. For these values, the confinement factors for the fundamental mode within the $\mathrm{Si}$ waveguide $\left(\Gamma_{\mathrm{Si}}\right)$ and within the MQW active regions $\left(\Gamma_{\mathrm{MQW}}\right)$ are more than $70 \%$ and $3 \%$, respectively, in a range of BCB bonding layer thicknesses between $20 \mathrm{~nm}$ and $120 \mathrm{~nm}$, as shown in Figure $2 \mathrm{~b}$. In order not to excite higherorder modes, current injection is confined using proton implantation in the lateral sections of the mesa.

\section{Device fabrication}

Device fabrication starts with the bonding of an unprocessed III-V die on a SOI die with the rib waveguides, using BCB. After the bonding, the III-V die is further processed to create a hybrid structure, as presented in Figure 1a. In our approach, BCB is spin-coated on a patterned SOI die, after which the III-V die is brought into contact with it and the $\mathrm{BCB}$ is cured. For this purpose, we developed a die-to-die bonding process for achieving ultra-thin $(\sim 50 \mathrm{~nm})$ $\mathrm{BCB}$ bonding layers [7]. A SEM image of the cross-section of the III-V die bonded on top of the SOI waveguide, using this procedure is illustrated in Figure 1b. The advantage of this process is that it can be scaled up to a multiple die-to-wafer bonding procedure and it is tolerant to surface imperfections. Due to the good planarization properties of BCB this approach allows bonding of very small III-V dies on integrated photonic circuits with rich surface topography, which is an additional advantage in achieving high-level integration in photonic circuits.

After the bonding, the InP substrate is removed by combination of grinding and wet-etching. Processing of a III$\mathrm{V}$ die starts with the definition of III-V islands, containing individual lasers, by using contact lithography and wet etching techniques. Mesa structures are fabricated combining dry etching and wet etching processes, after which Ntype metallization is performed. In the next processing step, BCB is spin-coated on the sample and cured, providing a physical protection and electrical isolation between the electrodes. Dry etching is used to make windows in a BCB covering layer for N-type and P-type contacts. P-type metallization is performed by sputtering a thin Ti layer, followed by the deposition of a $1.2 \mu \mathrm{m}$-thick Au layer. P-type electrodes serve as the mask for subsequent proton implantation which is used to locally increase electrical resistivity in the lateral regions of the mesa and confine the electrical current to the central region of the mesa. The width of the central P-type electrode, which allows the formation of the carrier injection channel, is $3 \mu \mathrm{m}$.

In the final processing step, gold is deposited on the electric contacts. In the end, individual devices are cleaved (or alternatively, diced and polished) and tested.

\section{Results}

The cleaved devices are tested with DC and pulsed current sources. The output power of the laser is measured at one facet of the device using a large-area photodetector positioned in close proximity to the facet, so that virtually all the 


\section{IWC3.pdf}

emitted optical power is detected. At this stage, testing is performed at room temperature and no active temperature control scheme is used. The measured I-V and L-I plots are given in Figure 3a). The turn-on voltage is $0.9 \mathrm{~V}$, the series resistance is $5.5 \Omega$ and the threshold current is $65 \mathrm{~mA}$. The device length is $900 \mu \mathrm{m}$ and the width of the current injection channel $\sim 3 \mu \mathrm{m}$, which gives a threshold current density of $2.41 \mathrm{kA} / \mathrm{cm}^{2}$. Maximum optical power in $\mathrm{CW}$ regime is $3.07 \mathrm{~mW}$, while the slope efficiency is $\sim 0.06 \mathrm{~W} / \mathrm{A}$.

The optical spectrum of the device is measured by coupling the output light into a single-mode fiber connected to an optical spectrum analyzer. Optical spectra of the laser at $100 \mathrm{~mA}$ and $130 \mathrm{~mA}$ injecting currents are presented in Figure $3 \mathrm{~b}$. Longitudinal modes, characteristic of Fabry-Perot lasers are clearly visible. The mode spacing is measured to be around $0.29 \mathrm{~nm}$, which corresponds to the group refractive index of 3.34 . With the increase in current from $100 \mathrm{~mA}$ to $130 \mathrm{~mA}$, the center wavelength shifts from $1317 \mathrm{~nm}$ to $1320.5 \mathrm{~nm}$.
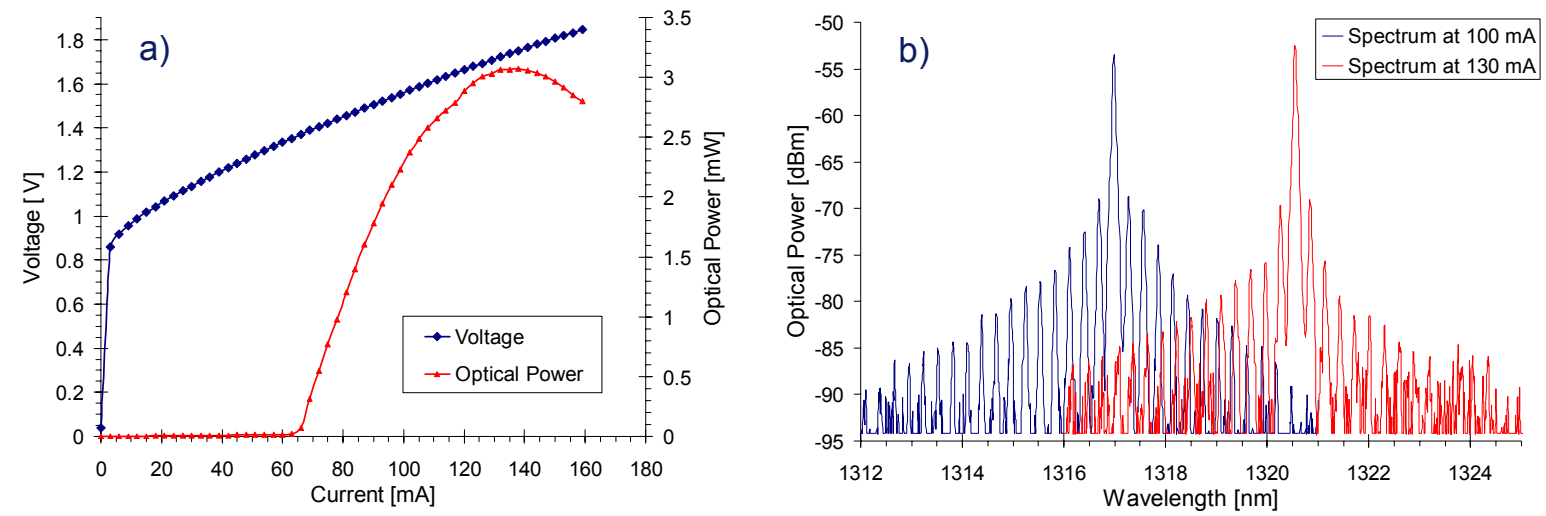

Figure 3. a) V-I and L-I plot of the hybrid laser, tested in CW regime; b) Optical spectra of the hybrid laser at $100 \mathrm{~mA}$ and $130 \mathrm{~mA}$ driving currents.

Limited pulsed testing of the lasers have been performed and they show the threshold current remains around 65 $\mathrm{mA}$, but the characteristic thermal rollover is not observed for current amplitudes less than $200 \mathrm{~mA}$. Further tests in the pulse regime will be discussed at the conference.

\section{Conclusions}

We have demonstrated evanescent hybrid III-V/silicon, Fabry-Perot lasers, based on BCB bonding. A threshold current of $65 \mathrm{~mA}$ was measured, corresponding to $2.4 \mathrm{kA} / \mathrm{cm}^{2}$ threshold current density. A maximum optical power of $3 \mathrm{~mW}$ was demonstrated in CW regime, with a slope efficiency of $0.06 \mathrm{~W} / \mathrm{A}$.

This evanescent hybrid laser was fabricated via a specially developed die-to-die BCB bonding technique, which offers more relaxed bonding conditions compared to molecular bonding, while enabling sufficiently thin bonding layers. Furthermore, it offers the prospects for development of a multiple die-to-wafer BCB bonding technique which would allow large-scale integration of hybrid lasers on wafers with photonic integrated circuits.

\section{Acknowledgements}

This work was supported by a grant from Intel Corporation. The authors wish to thank Hanan Bar from Numonyx, for fabrication of SOI wafers, and Liesbet Van Landschoot and Steven Verstuyft from the Photonics Research Group at Ghent University for SEM imaging and technical assistance in the cleanroom work.

\section{References}

[1] A.W. Fang, H. Park, R. Jones, O. Cohen, M. J. Paniccia, and J. E. Bowers, "A Continuous-Wave Hybrid AlGaInAs-Silicon Evanescent Laser," IEEE Photonics Technology Letters, 18(10), 1143-1145 (2006).

[2] Hsu-Hao Chang, A. W. Fang, M. N. Sysak, H. Park, R. Jones, O. Cohen, O. Raday, M. J. Paniccia, and J. E. Bowers, "1310nm silicon evanescent laser," Opt. Express, 15(18), 11466-11470 (2007).

[3] X. Sun, A. Zadok, M. J. Shearn, K. A. Diest, A. Ghaffari, H. A. Atwater, A. Scherer, and A. Yariv, "Electrically pumped hybrid evanescent Si/InGaAsP lasers," Opt. Lett., 34(9), 1345-1347 (2009).

[4] A. W. Fang, B. R. Koch, R. Jones, E. Lively, D. Liang, Y.-H. Kuo, and J. E. Bowers, "A Distributed Bragg Reflector Silicon Evanescent Laser," IEEE Photonics Technology Letters, 20(20), 1667-1669 (2008).

[5] A.W. Fang, E. Lively, Y. Kuo, D. Liang, and J. E. Bowers, "A distributed feedback silicon evanescent laser," Opt. Express, 16(7), 4413-4419 (2008).

[6] T. Hong, G. Ran, T. Chen, J. Pan, W. Chen, Y. Wang, Y. Cheng, S. Liang, L. Zhao, L. Yin, J. Zhang, W. Wang, and G. Qin, "A SelectiveArea Metal Bonding InGaAsP-Si Laser," IEEE Photonic Tech. L., 22(15), 1141-1143 (2010).

[7] S. Stankovic, D. Van Thourhout, G. Roelkens, R. Jones, J. Heck, M. Sysak, "Die-to-die adhesive bonding for evanescently-coupled photonic devices", ECS Transactions, 33(4), 411-420 (2010). 